\title{
AVM-Workshops bis Ende April 1992
}

Weitere Veranstaltungen werden in der nächsten Ausgabe der Zeitschrift bekanntgegeben. 13.-15.3.1992:

Psychopharmaka im Rahmen eines integrativen Therapiemodells (Dr. Rudolf Karazman) Information und Anmeldung: Karin Lettner, Institut für Psychologie der Universität Salzburg, Hellbrunner Straße 34, 5020 Salzburg, Tel. 0661/8044/5150.

21.-22.3.1992:

Kognitive Verhaltenstherapie bei Depressionen (Dr. Sevin Cayiroglu) Information und Anmeldung: Eveline Leitl, Kahlenberger Straße 7-9/2/9, 1190 Wien, Tel. 0222/374836

28.-29.3.1992:

Paradoxe Methoden in der Verhaltenstherapie - Praxiskurs III (Univ. Prof. Dr. Peter Fiedler) Information und Anmeldung: Georg Stuber, Eduard-Keil-Gasse 92, 8041 Graz, Tel. 0316/4612852.

4.-5.4.1992:

Training der sozialen Kompetenz (Dr. Berthold Judendorfer) Information und Anmeldung: Mag. Andrea Fahlböck, Wobischstraße la, 9500 Villach, Tel. 042 42/4 2308 oder 042 82/22 20/59.

4.-5.4.1992:

Integrierte Behandlung von akuten Angstanfällen (Panikattacken und Agoraphobie) (Dipl.Psych.

Jürgen Markgraf, Dipl.-Psych. Silvia Schneider)

Information und Anmeldung: Karin Lettner, Institut für Psychologie der Universität Salzburg, Hellbrunner Straße 34, 5020 Salzburg, Tel. 0662/8044/5150.

25.4.-6.5.1992:

Verhaltenstherapeutische Methoden der Schmerztherapie (Dr. Verena Günther)

Information und Anmeldung: Mag. Andrea Fahlböck, Wobischstraße la, 9500 Villach, Tel. 042 42/4 2308 oder 042 82/22 20/59.

Termin noch offen:

Selbsterfahrungsgruppe (Univ.-Doz. Dr. Herbert Mackinger)

Information und Anmeldung: Univ.-Doz. Dr. Herbert Mackinger, Wolfauweg 1, 5101 Bergheim, Tel. 52598.

Mitteilungen der Verbände

Kontaktadressen:

AVM Vereinssitz:

Dr. Anton Laireiter, Institut für Psychologie der Universität

Hellbrunner Straße 34

A-5020 Salzburg

Tel. (0043)662/8044/5122 
Geschäftsstelle:

Dr. Mercedes Zsifovics

Institut für Organisationspsychologie

Sigmund-Haffner-Gasse 18/3

A-5020 Salzburg

Tel. (0043)662/8044/6632 